\title{
Caracterización de pacientes con trauma esplénico atendidos en un hospital de tercer nivel entre enero de 2000 y diciembre de 2017
}

\author{
Characteristics of splenic trauma patients treated at a level III hospital \\ between January 2000 and December 2017
}

\author{
Gabriela Evers ${ }^{1} \mathbb{D}$, Rolando Medina ${ }^{2} \mathbb{D}$, Juan Sanjuán ${ }^{3} \mathbb{D}$, Sebastián Serrano ${ }^{4} \mathbb{D}$, \\ María Alejandra Rodríguez ${ }^{5}$ D, Gabriel Sánchez ${ }^{6}$ (D)
}

\begin{abstract}
Médica, residente de Cirugía General, Universidad Surcolombiana, Neiva, Colombia.
Médico, coordinador de Cirugía General, Universidad Surcolombiana; Cirugía General y Bariátrica, Soporte Nutricional, Hospital Hernando Moncaleano Perdomo, Neiva, Colombia.

3 Director CYTRA, Grupo de Investigación, Universidad Surcolombiana; cirugía general, Hospital Hernando Moncaleano Perdomo, Neiva, Colombia.

4 Médico interno, Universidad Surcolombiana, Neiva, Colombia.

5 Medica, Universidad Surcolombiana, Neiva, Colombia.

6 Médico interno, Universidad Surcolombiana, Neiva, Colombia.
\end{abstract}

\section{Resumen}

Introducción. Es frecuente (46 \%) que la lesión esplénica se acompañe de otra lesión visceral concomitante, con una alta tasa de morbimortalidad. La evolución de las técnicas para el abordaje de estas lesiones incluye procedimientos quirúrgicos, intervencionistas y de manejo expectante.

Métodos. Se trata de un estudio con cohorte única retrospectiva y observacional. Se incluyeron pacientes mayores de I3 años de edad con trauma esplénico, atendidos entre enero de 2000 y diciembre de 20I7. Se describieron las características relacionadas con el proceso de atención.

Resultados. Se identificaron II6 pacientes con trauma esplénico, el 85,2 \% de ellos hombres, con una edad promedio de 26 años. El 75,9 \% de los pacientes presentaba lesiones concomitantes; las más frecuentes fueron de diafragma (3I,O \%), de hígado (I7,2 \%) y de riñón (II,2 \%). Los mecanismos de lesión más frecuentes fueron por arma corto-punzante $(29,3 \%)$, por arma de fuego $(22,4 \%)$ y por accidentes de tránsito $(22,4 \%)$. La gravedad fue clasificada como de grado $\mathrm{V}$ en el 24,I \% de los pacientes y de grado I en el 23,3\%. Se practicó esplenectomía total en el 39,7 \% de los pacientes y el 15,2 \% fue manejado de forma conservadora. La mortalidad fue del 4,3\%, tres casos en el posoperatorio inmediato (menos de 24 horas) y dos en la primera semana posoperatoria.

Fecha de recibido: 31/06/2019 - Fecha de aceptación: 21/08/2019

Correspondencia: Gabriela Evers-Sáenz, Calle 56 №1W-99, Neiva, Colombia Teléfono: (317) 437-6803

Correo electrónico: gabriela_evers@msn.com

Citar como: Evers G, Medina R, Sanjuán J, Serrano S, Rodríguez MA, Sánchez G. Caracterización de pacientes con trauma esplénico atendidos en un hospital de tercer nivel entre enero de 2000 y diciembre de 2017. Rev Colomb Cir. 2020;35:464-71. https://doi.org/10.30944/20117582.729

Este es un artículo de acceso abierto bajo una Licencia Creative Commons - BY-NC-ND https://creativecommons.org/licenses/by-ncnd/4.0/deed.es 
Discusión. El manejo conservador de las lesiones esplénicas de poca gravedad es una estrategia segura y efectiva, con una tasa de fracaso de menos del $5 \%$. El abordaje quirúrgico se reserva para lesiones de mayor gravedad y su relación con otros órganos lesionados, con una mayor morbimortalidad (63\%).

Palabras clave: traumatismos abdominales; bazo; rotura del bazo; esplenectomía; tratamiento conservador; procedimientos endovasculares.

\begin{abstract}
Introduction. Splenic injuries are usually accompanied by another concomitant visceral lesion (46\%), with a high rate of morbidity and mortality. The evolution of techniques for approaching these injuries includes surgical, interventional and expectant management procedures.

Methods. Retrospective, observational single cohort study. Patients older than I3 years of age with splenic trauma who were treated between January 2010 and December 2017 were included. The characteristics related to the care process were described.

Results. II6 patients with splenic trauma were identified, $85,2 \%$ were men and the average age was 26 years. Concomitant lesions were identified in 75,9\% of patients, the most common were diaphragm (3I \%), liver (I7,2 \%), and kidney (II,2 \%). The most common mechanisms of injury were stab wounds (29,3\%), gunshot wounds (22,4 \%), and motor vehicle collisions (22,4 \%). Severity was classified as grade V in $24, \mathrm{I} \%$ of patients and grade I in $23,3 \%$. Total splenectomy was performed in $39,7 \%$ of patients and $\mathrm{I} 5,2 \%$ was conservatively managed. Mortality was $4,3 \%$, three cases in the immediate postoperative period (within 24 hours) and two in the first week postoperative week.

Discussion. Conservative management of low-grade splenic injuries is a safe and effective strategy, with a failure rate of less than $5 \%$. Surgical approach is reserved for injuries with greater severity and their relationship with other injured organs, with greater morbidity and mortality (63\%).
\end{abstract}

Key words: abdominal injuries; spleen; splenic rupture; splenectomy; conservative treatment; endovascular procedures.

\section{Introducción}

El trauma abdominal puede ser clasificado en dos grandes grupos, cerrados o abiertos. El abdomen es la cuarta zona corporal más afectada en los traumas cerrados, necesariamente con compromiso de sus estructuras hasta en el $15 \%$ de las víctimas con trauma múltiple ${ }^{\mathrm{I}, 2}$. Por esta razón, el trauma abdominal se convierte en la segunda causa de muerte por trauma cerrado, en la mayoría de los casos por hemorragia abdominal con posterior falla multiorgánica o sepsis ${ }^{1,2}$. El bazo es el órgano más frecuentemente lesionado, hasta en el $46 \%$ de los casos de trauma cerrado 3,4; es un órgano que, por su abundante irrigación sanguínea y su localización, es el origen de hemorragias intraabdominales mayores ${ }^{3,4}$. Se registran mortalidades hasta del $38 \%$ en los accidentes de tránsito, seguido de las caídas de diferentes alturas. Las heridas penetrantes son menos comunes (5 a I4 \%) ${ }^{5}$.

Es poco común que se presente una lesión única del bazo por un trauma abdominal, específicamente uno contuso, ya que casi siempre se lesionan también otros órganos. El trauma esplénico se acompaña de lesiones en el tórax en el 37 al $77 \%$ de los casos, de lesiones craneoencefálicas en el 46 al $59 \%$ y de lesiones hepáticas en el 3i $\%{ }^{5}$.

El enfoque para el manejo de los pacientes con traumatismos abdominales mayores, se encuentra estandarizado en protocolos ya establecidos por el Advance Trauma Life Support (ATLS ); el principio fundamental planteado es identificar y tratar las lesiones que amenazan la vida de manera inmediata ${ }^{6,7}$. Para esto, la tomografía com- 
putarizada con contraste se considera el método diagnóstico estándar, ya que revela la presencia de sangrado activo lo cual constituye el principal factor pronóstico ${ }^{8,9}$, con una sensibilidad del $76 \%$, una especificidad del $90 \%$ y una precisión del $83 \%$ para detectar la lesión vascular esplénica ${ }^{\mathrm{I}}$. Esto ha contribuido a que el trauma esplénico contuso se maneje de forma no quirúr-

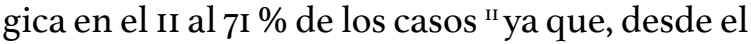
siglo pasado, su tratamiento ha cambiado: dejó de ser quirúrgico para todas las lesiones y, en la actualidad, se está usando el manejo expectante y de observación ${ }^{12}$. La mortalidad total es del $26 \%$ en los pacientes sometidos a una intervención quirúrgica inmediata, del $4 \%$ en los que el manejo no quirúrgico es exitoso, y del $\mathrm{I} 6,5 \%$ en quienes falla el tratamiento no quirúrgico ${ }^{13}$.

\section{Métodos}

Se llevó a cabo un estudio observacional y descriptivo de una cohorte única retrospectiva. El Hospital Universitario de Neiva corresponde a un centro académico surcolombiano de referencia y de nivel III de atención.

Se consideró un tamaño de muestra de conveniencia no probabilística. Se revisaron los registros médicos relacionados con procedimientos quirúrgicos o diagnósticos de lesión esplénica entre el año 2000 y el 20I7, utilizando los códigos diagnósticos CIEıo consignados en la historia clínica y los informes quirúrgicos que hacían posible el diagnóstico de trauma esplénico. Los pacientes se clasificaron según los cinco diferentes grados establecidos por la American Association for the Surgery of Trauma (AAST) ${ }^{\mathrm{I}}$.

\section{Criterios de selección}

Se incluyeron los pacientes mayores de 13 años de edad que ingresaron al servicio de urgencias y en quienes se diagnosticó algún grado de compromiso esplénico. Se excluyeron los pacientes sin información sobre la estancia hospitalaria o las características del tratamiento de la lesión esplénica, y aquellos que fueron intervenidos quirúrgicamente en una institución diferente al Hospital Universitario de Neiva.
Se recopiló la información sobre las características sociodemográficas y clínicas, la necesidad de intervención quirúrgica, las complicaciones y la mortalidad al egreso hospitalario. Esta información se almacenó en el paquete estadístico Excel $^{\circledR}$.

\section{Análisis estadístico}

Se llevó a cabo un análisis estadístico descriptivo; para las variables categóricas, se determinaron las frecuencias absolutas (n) y relativas (\%), la mediana continua (Med) y el rango intercuartílico (RIC).

\section{Resultados}

Durante el periodo de enero de 2000 a diciembre de 20I7, en el Hospital Universitario de Neiva se identificaron II6 pacientes en quienes se diagnosticó trauma esplénico mediante algún tipo de estudio o sometidos a alguna intervención quirúrgica como consecuencia de este.

La edad promedio fue de 26 años. Hubo mayor prevalencia del trauma esplénico en la población masculina, 98 (85,2\%), y fue más frecuente en personas sin cubrimiento de seguridad social y en las del régimen subsidiado. La mayoría de los pacientes, 94 de ellos, tuvieron que ser sometidos a algún tipo de procedimiento quirúrgico, mientras que solo a I8 no se les practicó ningún procedimiento invasivo.

Las heridas por arma corto-punzante fueron el mecanismo de trauma más común, en 34 $(29,3 \%)$ de los pacientes, seguidas por las heridas por arma de fuego y los accidentes de tránsito, ambos en $26(22,4 \%)$ pacientes; los traumas menos frecuentes fueron otros tipos de golpes contundentes, incluidos los casos de caídas de altura (figura I).

De todas las intervenciones quirúrgicas requeridas, la principal fue la esplenectomía total, practicada en $46(39,7 \%)$ de pacientes, seguida de la esplenorrafia en 39 (33,6 \%). En I5 (I8 \%) casos no fue necesaria ninguna intervención en el bazo, pues no presentó sangrado activo (figura 2).

De acuerdo con la clasificación de la AAST de la gravedad de la alteración anatómica del bazo 


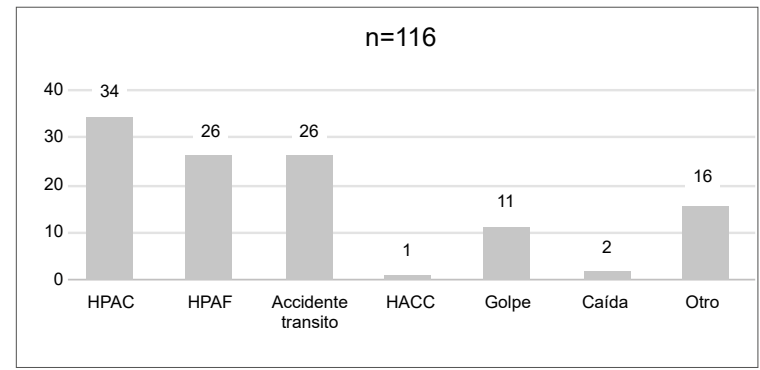

Figura 1. Mecanismos de lesión en los pacientes con trauma esplénico

HACP: herida por arma corto-punzante. HPAF: herida por proyectil de arma de fuego. HACC: herida por arma corto-contundente.

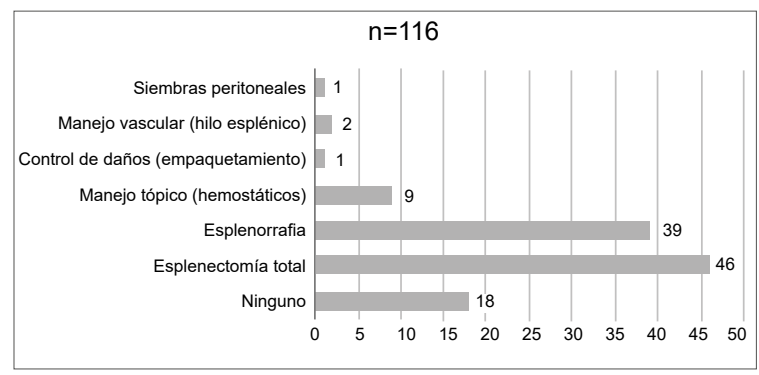

Figura 2. Tipos de procedimientos realizados a los pacientes con trauma esplénico y según la Abreviated Injury Scale (AIS), en este estudio los grados de trauma esplénico presentados con mayor frecuencia fueron el grado $\mathrm{V}$ en $28(24, \mathrm{I} \%)$ pacientes, el grado I en $27(23,3 \%)$ y el grado II en $26(22,4 \%)$. En la tabla I se muestran los procedimientos quirúrgicos practicados en relación con esta clasificación.

Se documenta que la intervención quirúrgica radical con esplenectomía total se practicó en los casos más graves, mientras que los tratamientos más conservadores fueron posibles en los menos graves: además, en I2 $(66,7 \%)$ con el menor grado de trauma esplénico, muy probablemente un hematoma o una pequeña laceración sin sangrado, no hubo intervención alguna sobre el bazo.

Es frecuente que el trauma esplénico se asocie con otras lesiones intraabdominales concomitantes, lo cual sucedió en $87(75,9 \%)$ pacientes (tabla 2). En su orden de frecuencia como hallazgos intraoperatorios abdominales entre los II6 pacientes, las lesiones diafragmáticas fueron de grado III en I6 (I3,8\%) pacientes y de grado II en I5 (I2,9 \%); en segundo lugar, las lesiones hepáticas fueron de grado I en $8(6,9 \%)$ pacientes

Tabla 1. Relación del grado de lesión esplénica con el tipo de procedimiento

\begin{tabular}{cccccc}
\hline Grado & Total & Esplenectomía & Esplenorrafia & Hemostáticos & Ninguno \\
\hline I & 27 & 1 & 8 & 5 & 12 \\
II & 26 & 3 & 18 & 1 & 4 \\
III & 21 & 7 & 9 & 3 & 1 \\
IV & 14 & 11 & 3 & 0 & 0 \\
V & 28 & 24 & 1 & 0 & 1 \\
\hline
\end{tabular}

Tabla 2. Lesiones asociadas al trauma esplénico

\begin{tabular}{lclc}
\hline \multicolumn{2}{l}{ Lesiones abdominales } & \multicolumn{2}{l}{ Lesiones extraabdominales } \\
\hline Órgano & $\mathbf{N ~ ( \% )}$ & Órgano & $\mathbf{N ~ ( \% )}$ \\
\hline Diafragma & $36(31 \%)$ & Lesión pleural & $26(22,4 \%)$ \\
Hígado & $20(17,2 \%)$ & Hemotórax & $17(14,7 \%)$ \\
Riñón & $13(11,2 \%)$ & Neumotórax & $4(3,5 \%)$ \\
Estómago & $12(10,3 \%)$ & Hemoneumotórax unilateral & $3(2,6 \%)$ \\
Colon & $9(7,8 \%)$ & Hemoneumotórax bilateral & $2(1,7 \%)$ \\
Intestino delgado & $9(7,8 \%)$ & Pulmón & $2(1,7 \%)$ \\
Páncreas & $9(7,8 \%)$ & Corazón & $2(1,7 \%)$ \\
Duodeno & $2(1,7 \%)$ & Grandes vasos & $1(0,9 \%)$ \\
Grandes vasos & $4(3,4 \%)$ & Retroperitoneo & $16(13,8 \%)$ \\
\hline
\end{tabular}


y, de grado II o III, en 5 (4,3\%); en tercer lugar, las lesiones renales fueron de grado I en $6(5,2 \%)$ pacientes y, por último, las del estómago fueron de grado III en $6(5,2 \%)$ individuos (tabla 3 ).

De los II6 pacientes con trauma esplénico durante el periodo de estudio, $5(4,3 \%)$ murieron, 3 $(2,6 \%)$ durante la cirugía o antes de transcurridas las primeras 24 horas, y $2(\mathrm{I}, 7 \%)$ durante la primera semana del posoperatorio (tabla 4).

\section{Discusión}

En este estudio se caracterizaron los casos de trauma esplénico atendidos en un hospital de tercer nivel, donde el trauma es uno de los más frecuentes motivos de consulta. Se hizo considerando los diferentes mecanismos de trauma abdominal y correlacionándolos con los rangos de edad. Los datos obtenidos se compararon con lo descrito en la literatura científica y se obtuvieron comparaciones muy valiosas y representativas.

Es muy común que el bazo se lesione durante un trauma abdominal contundente cerrado, cuya causa más frecuente son los accidentes de tránsito, con mortalidades de hasta el $38 \%$ 5 . En este trabajo, el trauma cerrado ocasionó la lesión esplénica en 33,6 \% (39/II6) de los pacientes. Dentro de este grupo, la principal causa fueron los accidentes de tránsito, ocurridos en $26(22,4 \%)$ de los pacientes, seguidos del trauma contundente y, en último lugar, las caídas, lo cual concuerda con lo descrito en la literatura científica.

Se debe tener extrema precaución con los politraumatismos en accidentes de tránsito, ya que esta es la causa más común de muerte previsible en pacientes con traumas abdominales. Además, se encontró una mortalidad de 4,3\% (5/II6), muy

Tabla 3. Clasificación AIS de los órganos intraabdominales comprometidos concomitantemente

\begin{tabular}{cccccccccc}
\hline $\begin{array}{c}\text { Grado de } \\
\text { lesión }\end{array}$ & $\begin{array}{c}\text { Diafragma } \\
\mathbf{n}(\%)\end{array}$ & $\begin{array}{c}\text { Hígado } \\
\mathbf{n}(\%)\end{array}$ & $\begin{array}{c}\text { Riñón } \\
\mathbf{n}(\%)\end{array}$ & $\begin{array}{c}\text { Estómago } \\
\mathbf{n}(\%)\end{array}$ & $\begin{array}{c}\text { Colon } \\
\mathbf{n}(\%)\end{array}$ & $\begin{array}{c}\text { Intestino } \\
\text { delgado } \\
\mathbf{n}(\%)\end{array}$ & $\begin{array}{c}\text { Páncreas } \\
\mathbf{n}(\%)\end{array}$ & $\begin{array}{c}\text { Duodeno } \\
\mathbf{n}(\%)\end{array}$ & $\begin{array}{c}\text { Grandes } \\
\text { vasos } \\
\mathbf{n}(\%)\end{array}$ \\
\hline I & $4(3,5)$ & $8(6,9)$ & $6(5,2)$ & $3(2,6)$ & $4(3,5)$ & $1(0,9)$ & $3(2,6)$ & $1(0,9)$ & $1(0,9)$ \\
II & $15(12,9)$ & $5(4,3)$ & $1(0,9)$ & $1(0,9)$ & $2(1,7)$ & $3(2,6)$ & $1(0,9)$ & 0 & $1(0,9)$ \\
II & $16(13,8)$ & $5(4,3)$ & $3(2,6)$ & $6(5,2)$ & $3(2,6)$ & $5(4,3)$ & $2(1,7)$ & $1(0,9)$ & 0 \\
IV & $1(0,9)$ & $1(0,9)$ & $2(1,7)$ & $2(1,7)$ & 0 & 0 & $2(1,7)$ & 0 & 0 \\
V & 0 & $1(0,9)$ & $1(0,9)$ & 0 & 0 & 0 & $1(0,9)$ & 0 & $2(1,7)$ \\
\hline
\end{tabular}

Tabla 4. Mortalidad

\begin{tabular}{|c|c|c|c|c|}
\hline & $\begin{array}{l}\text { Mecanismo } \\
\text { del trauma }\end{array}$ & $\begin{array}{c}\text { Grado de trauma } \\
\text { esplénico }\end{array}$ & Lesiones asociadas & $\begin{array}{l}\text { Procedimiento } \\
\text { quirúrgico }\end{array}$ \\
\hline \multirow[t]{3}{*}{$\begin{array}{l}\text { Antes de } 24 \text { horas } \\
\text { del posoperatorio }\end{array}$} & Accidente de tránsito & $\mathrm{I}$ & $\begin{array}{l}\text { Hematoma retroperitoneal de las } \\
\text { tres zonas + lesión diafragmática } \\
\text { de grado IV + } 300 \mathrm{ml} \text { hemope- } \\
\text { ritoneo }\end{array}$ & $\begin{array}{l}\text { Control de daños con } \\
\text { empaquetamiento }\end{array}$ \\
\hline & Accidente de tránsito & IV & Hemoperitoneo $100 \mathrm{ml}$ & Esplenectomía \\
\hline & Accidente de tránsito & $\mathrm{V}$ & $\begin{array}{l}\text { Hemoperitoneo } 2.000 \mathrm{ml}+\text { lesión } \\
\text { de grado } \mathrm{V} \text { de grandes vasos } \\
\text { intraabdominales + hematoma } \\
\text { retroperitoneal + lesión hepática } \\
\text { de grado } \mathrm{V}\end{array}$ & Esplenectomía \\
\hline \multirow[t]{2}{*}{$\begin{array}{l}\text { Durante la primera } \\
\text { semana del } \\
\text { posoperatorio }\end{array}$} & Accidente de tránsito & $\mathrm{V}$ & $\begin{array}{l}\text { Hemoperitoneo de } 2.500 \mathrm{ml} \\
\text { + lesión hepática de grado I + } \\
\text { hemotórax unilateral } \\
\text { Trauma craneoencefálico }\end{array}$ & Esplenectomía \\
\hline & $\begin{array}{l}\text { Herida por arma de } \\
\text { fuego }\end{array}$ & 1 & Sin otras lesiones asociadas & Esplenorrafia \\
\hline
\end{tabular}


por debajo de la registrada por otros autores. En lo que sí concuerda es con el mecanismo de trauma, por lo que se le asocia esta mortalidad, ya sea por la lesión del bazo, o por otras lesiones graves concomitantes.

En la literatura científica, las heridas penetrantes esplénicas tienen una frecuencia del $5 \mathrm{al}$ I $4 \%{ }^{5}$. Este estudio arrojó resultados completamente contrarios, ya que se evidenció que más de la mitad (6o/II6, 5I,7\%) de los pacientes presentaron trauma abdominal penetrante (por arma corto-punzante o por arma de fuego) como causa del trauma esplénico.

Las complicaciones del trauma esplénico se relacionan con la gravedad de la lesión, con lesiones asociadas, con fallas diagnósticas o con tratamientos inadecuados. En el estudio se lograron identificar las lesiones asociadas más frecuentes y los grados de trauma esplénico que condicionan una decisión quirúrgica. Se sabe que las heridas olvidadas o los diagnósticos y tratamientos tardíos llevan por sí mismos a altas tasas de mortalidad, por lo que se debe siempre garantizar una atención rápida y oportuna para evitar complicaciones.

Con respecto a las lesiones esplénicas, se encontró que, en la mayoría de los pacientes con tratamiento invasivo radical con esplenectomía total, el trauma esplénico fue de grado V o IV e, incluso, algunos de grado III. Los tratamientos más conservadores, como la esplenorrafia, se usaron en la mayoría de los traumas menos graves, grado I o II, y no se utilizaron en los traumas más graves.

Como ya se mencionó, es muy poco común que el bazo sea el único órgano lesionado después de un trauma; casi siempre coexisten lesiones torácicas ( 37 a $77 \%$ ), craneoencefálicas (46 a $59 \%$ ) o hepáticas $(3 \mathrm{I} \%)^{5}$, como se corroboró en este estudio.

Desde el siglo pasado, el tratamiento del trauma del bazo ha ido cambiando, de ser quirúrgico en todos los $\operatorname{casos}^{8,15,16}$, a lo que se está haciendo en la actualidad, que es el manejo expectante y de observación ${ }^{3,13,17,18}$. Anteriormente, el tratamiento estándar para las lesiones esplénicas me- nores era la esplenectomía temprana, con el fin de evitar la hemorragia fatal. Sin embargo, poco a poco y gracias a un mejor conocimiento de las funciones del bazo, más cirujanos han preferido un manejo conservador, sea una esplenectomía parcial o una intervención no quirúrgica cuando sea posible. Las ventajas de este tipo de manejo incluyen menores costos hospitalarios, egresos hospitalarios tempranos, menos complicaciones intraabdominales y reducción de las tasas de transfusión, asociadas con una mejoría general en la mortalidad de estos pacientes ${ }^{12}$. La evaluación y el tratamiento de las lesiones esplénicas se ha desarrollado a lo largo de varias décadas, como resultado del avance en el campo de las imágenes diagnósticas ${ }^{19-21}$ y la experiencia acumulada sobre los diferentes tipos de tratamiento, quirúrgico versus conservador.

El manejo no quirúrgico de las lesiones esplénicas, en especial por trauma contundente, fue abordado por primera vez por la Eastern Association for the Surgery of Trauma (EAST) en las Practice Management Guidelines for the Nonoperative Management of Blunt Injury to the Liver and Spleen, publicadas en $2003^{22}$. En ellas se acepta que el paciente con estabilidad hemodinámica y sin otras lesiones abdominales que requieran intervención, sea manejado con monitorización continua. Cuanto mayor sea la gravedad de la lesión esplénica, mayor es la tasa de fracaso del tratamiento expectante.

Para las lesiones esplénicas de bajo grado (AAST I a III), la tasa de fracaso se mantiene por debajo del $5 \%$, pero, con un grado IV, aumenta a $23 \%$ y, con grado $\mathrm{V}$, a $63 \%{ }^{22,23}$. Los pacientes que con inestabilidad hemodinámica o con líquido libre intraabdominal demostrado, deben someterse a una laparotomía exploratoria inmediata, ya que, por lo general, se va a encontrar una lesión asociada de otro órgano que requiere corrección prioritaria, o se puede tratar de una lesión esplénica única causante de la inestabilidad del paciente; cualquiera que sea el hallazgo intraoperatorio, la indicación es la cirugía urgente ${ }^{24}$.

Con esta caracterización, se planteó la posibilidad de crear una guía propia del Hospital 
Hernando Moncaleano Perdomo, en la cual se incluyan los exámenes de tomografía computarizada, siempre y cuando las condiciones del paciente lo permitan, para tomar decisiones clínico-quirúrgicas y, en un futuro, fomentar el manejo conservador de las lesiones de bajo grado y limitar los tratamientos drásticos, como la esplenectomía, para los traumas de grados altos.

\section{Cumplimiento de normas éticas}

Consentimiento informado. Este estudio es una revisión retrospectiva de historias clínicas y, como tal, no hay necesidad de un consentimiento informado. El Comité de Ética Institucional aprobó el diseño y la metodología del estudio.

Declaración de conflicto de intereses. Los autores declaran que no tienen conflicto de intereses.

Fuentes de financiación. Recursos propios de los autores.

\section{Referencias}

I. Camacho Durán F, Garavito CE. Guías para manejo de urgencias. Tomo I: Grupo de Atencion de Emergencias y Desastres. Bogotá: Ministerio de Protección Social; 2005.

2. Castro-Rendón E. Manejo diagnóstico del trauma cerrado de abdomen en adultos. Guía de práctica clínica Hospital Provincial Neuquén. 20I6. Disponible en: https://archivo.saludneuquen.gob.ar/wp-content/ uploads/20I2/o9/40-GPC-trauma-abdomen-cerrado-HPN-20I6.pdf.

3. Yorkgitis BK. Primary care of the blunt splenic injured adult. Am J Med. 20I7;130:365.eI-365.e5. https://doi.org/Io.IoI6/j.amjmed.20I6.Io.oIo

4. Cirocchi R, Boselli C, Corsi A, Farinella E, Listorti C, Trastulli $\mathrm{S}$, et al. Is non-operative management safe and effective for all splenic blunt trauma? A systematic review. Crit Care. 2013;17:RI85. doi: I0.II86/ccI2868

5. Williamson J. Splenic injury: Diagnosis and management. Br J Hosp Med (London). 2015;76:204-29. https://doi.org/IO.I2968/hmed.20I5.76.4.204

6. Shoobridge JJ, Corcoran NM, Martin KA, Koukounaras J, Royce PL, Bultitude MF. Contemporary management of renal trauma. Rev Urol. 20II;13:65-72. https://doi.org/I0.3909/riuo5I2

7. American College of Surgeons. Soporte vital avanzado en trauma ATLS. 9th edition. Chicago: Advanced Trauma Life Support (ATLS); 2012.
8. Marmery H, Shanmuganathan K, Alexander MT, Mirvis SE. Optimization of selection for nonoperative management of blunt splenic injury: Comparison of MDCT grading systems. Am J Roentgenol. 2007;I89:I42I-7. https://doi.org/I0.22I4/AJR.07.2152

9. Musalar E, Ersel M, Akarca FK, Kıyan GS, Can Ö. The predictive value of biochemical parameters in evaluating patients with abdominal trauma: The new scoring system. Turkish J Emerg Med. 2017;17:48-55. https://doi.org/Io.Ior6/j.tjem.20I6.II.005

Io. Bradley MJ, O'Connor JV. Blunt splenic injury. Trauma. 2015;I7:I42-5. https://doi.org/Io.II77/I4604086I4565966

II. Hildebrand DR, Ross NP, Macvicar R, Frizelle FA, Watson AJM. Modern management of splenic trauma. $\mathrm{Br}$ Med J. 2OI4;348:I-7. https://doi.org/IO.II36/bmj.gI864

I2. Stassen NA, Bhullar I, Cheng JD, Crandall ML, Friese RS, Guillamondegui OD, et al. Selective nonoperative management of blunt splenic injury: An eastern association for the surgery of trauma practice management guideline. J Trauma Acute Care Surg. 2012;73:¿páginas? https://doi.org/I0.I097/TA.oboize3182702afc

13. Watson GA, Hoffman MK, Peitzman AB. Nonoperative management of blunt splenic injury: What is new? Eur J Trauma Emerg Surg. 2015;41:219-28. https://doi.org/ I0.IO07/s00068-0I5-0520-I

I4. Moore EE, Cogbill TH, Malangoni M, Jurkovich GJ, Champion HR. Scaling system for organ specific injuries. Disponible en: file:///C:/Users/Usuario/Downloads/InjuryScoringTables\%20(3).pdf

15. Sinha S, Raja SV, Lewis MH. Recent changes in the management of blunt splenic injury: Effect on splenic trauma patients and hospital implications. Ann R Coll Surg Engl. 2008;90:I09-I2. https://doi.org/I0.I308/003588408X242033

I6. Pachter HL, Guth AA, Hofstetter SR, Spencer FC. Changing patterns in the management of splenic trauma: The impact of nonoperative management. Ann Surg. 1998;227:708-9.

https://doi.org/I0.I097/00000658-199805000-000II

I7. Olthof DC, van der Vlies CH, Goslings JC. Evidence-based management and controversies in blunt splenic trauma. Curr Trauma Reports. 2017;3:32-7. https://doi.org/I0.I007/s40719-017-0074-2

I8. Yiannoullou P, Hall C, Newton K, Pearce L, Bouamra $\mathrm{O}$, Jenks T, et al. A review of the management of blunt splenic trauma in England and Wales: Have regional trauma networks influenced management strategies and outcomes? Ann R Coll Surg Engl. 2017;99:63-9. https://doi.org/IO.I308/rcsann.2016.0325

I9. Boutros SM, Nassef MA, Abd el-ghany AF. Blunt abdominal trauma: The role of focused abdominal sonography in assessment of organ injury and reducing the 
need for CT. Alexandria J Med. 2016;52:35-4I. https://doi.org/Io.IoI6/j.ajme.2015.02.00I

20. Hildebrand DR, Ross NP, Macvicar R, Frizelle F, Watson JM. Modern management of splenic trauma. $\mathrm{Br}$ Med J. 20I4;348:I-7. https://doi.org/IO.II36/bmj.gI864

2I. Soto JA, Anderson SW. Multidetector CT of blunt abdominal trauma. Radiology. 2012;265:678-93.

https://doi.org/IO.II48/radiol.I2I20354

22. Alonso M, Brathwaite C, García V, Patterson L, Scherer $\mathrm{T}$, Stafford $\mathrm{P}$, et al. Practice management guidelines for the nonoperative management of blunt injury to the liver and spleen. East Assoc Surg Trauma. 2003;43:I-32. https://doi.org/IO.I097/00005373-I997IIO00-000I4

23. Bittenbinder EN, Reed AB. Advances in renal intervention for trauma. Semin Vasc Surg. 2013;26:165-9. https://doi.org/IO.IO53/j.semvascsurg.20I4.06.0I2

24. Toro JP, Arango PA, Villegas MI, Morales CH, Echavarría A, Ortiz MM, et al. Trauma esplénico cerrado: predictores de la falla del manejo no operatorio. Rev Colomb Cir. 2014;29:204-I2. 